decided to study the incidence and clinical spectrum of APS in a general hospital.

Objectives To examine the spectrum of clinical manifestations of antiphospholipid antibody syndrome (APS) in the acute general hospital of the southern area of Israel.

Methods Clinical records of patients who were discharged with diagnosis of APS during the years 1990-2000 were reviewed.

Results A total of 22 patients fulfilled criteria of APS (accordingly to Asherson?s criteria, 1988) with 38 clinical events. Primary APS was found in 15 patients and secondary in 7 patients. There were 19 female and 3 male, mean age was 42.1 years (from 29 to 56). Most of the patients (20 out of 22) were diagnosed after 1995, showing increased awareness of this syndrome among the stuff.

16 thrombotic events occurred: 6 stroke (27\%), 4 acute myocardial infarction $(18 \%), 3$ venous thromboembolic event (13.6\%), 3 recurrent abortion (13.6\%). 32 nonthrombotic events were found: 8 headache (36.3\%), 6 thrombocytopenia (27\%), 3 skin rashes (13.6\%), 1 amaurosis fugax (4.5\%), 1 catastrophic APS (4.5\%) 1 progressive supranuclear pulsy (PSP) (4.5\%).

All patients suffering from acute myocardial infarction were patients with SLE, thus showing that APS is an additional risk factor for ischaemic heart disease in such patients.

Headache was an additional symptom in the patients, diagnosed as having APS - in all 6 patients with stroke, one with amaurosis fugax and one with venous thromboembolic event.

Patient with PSP features had magnetic resonance imaging of brain and blood test results characteristic to APS (our unpublished data).

Conclusion The study shows that APS is not an uncommon disease. It is important for the internist and the specialist to be aware of the clinical manifestations of APS. Its inclusion in the differential diagnosis of arterial or venous thrombosis with or without thrombocytopenia in patients is obligatory at the present time.

\section{FRI0178 FOLLOWUP OF PLATELET ACTIVATION IN A PATIENT WITH ANTIPHOSPHOLIPID SYNDROME UPON TIROFIBAN TREATMENT}

${ }^{1}$ A Perniok, ${ }^{1} \mathrm{~A}$ Messis, ${ }^{2} \mathrm{C}$ Specker, ${ }^{3} \mathrm{M}$ Siebler, ${ }^{2} \mathrm{M}$ Schneider. ${ }^{1}$ Department of Medicine II, University of Cologne, Cologne, Germany; ${ }^{2}$ Department of Rheumatology, University of Düsseldorf, Düsseldorf, Germany; ${ }^{3}$ Department of Neurology, University of Düsseldorf, Düsseldorf, Germany

\subsection{6/annrheumdis-2001.247}

Background Patients with primary or secondary antiphospholipid syndrome (APS) often suffer from stroke leading to high degree of disability. Though it is well known that phospholipid antibodies induce a procoagulatory scenario, antibody status does not well characterise patients at risk. Using transcranial Doppler ultrasound of intracerebral arteries we could detect microembolic signals correlating well with phospholipid antibody titer as well as with history of stroke. In these patients we could also demonstrate elevated levels of platelet activation markers (p-selectin) as well as increased platelet leukocyte aggregation. Tirofiban is a potent, highly selective and reversible inhibitor of platelet gpIIb/IIIa receptor which binds fibrinogen on platelet surface.

Objectives We treated a patient with primary APS and refractory transitory ischaemic attacks with tirofiban and detected microembolic signals as well as platelet activation markers and platelet-leukocyte aggregations (PLA).
Methods In fresh venous citrate-anticoagulated whole blood expression of gp Ib (CD41), gp IIb/IIIa (CD42b), fibrinogen binding and p-selectin (CD62p) was analysed using a flowcytometric double staining method. In parallel we detected PLA by determining percentage of platelet marker/leukocyte marker (CD42b/CD45) double posi-tive leukocytes.

Results Upon Tirofiban treatment we found a reduction of platelet activation markers ( $p$-selectin, fibrinogen binding, CD63). Under steady state conditions with tirofiban expression of platelet activation markers and platelet leukocyte interaction normalised completely compared to normal healthy donors. The morning after each infusion there was a rebound phenomenon, which could also be observed in platelet activation markers and PLA. This rebound phenomenon could be reproduced upon sequential administration of tirofiban in parallel with rate of microembolic events.

Conclusion In a patient with primary APS therapeutic intervention with tirofiban, a reversible inhibitor of platelet gpIIb/IIIa receptor, induced a clinical improvement of transitory ischaemic attacks. In parallel with clinical improvement there was a modulation of microembolic signals as well as platelet activation markers and PLA. Flowcytometric measurement of platelet activation can be a useful tool in monitoring platelet hyperreactivity in APS upon therapeutic intervention.

\section{FRI0179 NAILFOLD CAPILLAROSCOPY IN SYSTEMIC LUPUS ERYTHEMATOSUS PATIENTS WITH AND WITHOUT ANTIPHOSPHOLIPID SYNDROME}

V Riccieri, A Spadaro, T Rinaldi, R Scrivo, F Ceccarelli, G Valesini. Cattedra Di Reumatologia - Dipartimento Di Terapia Medica, Università Di Roma "La Sapienza", Rome, Italy

\subsection{6/annrheumdis-2001.248}

Background Nailfold capillaroscopy is able to evaluate microcirculatory damage in many different diseases and a high prevalence of capillary abnormalities has been reported in systemic lupus erythematosus (SLE).

Objectives Considering that the vascular involvement is of primary importance in antiphospholipid syndrome (APS), it can be of interest to examine the capillaroscopic features of those SLE patients having secondary APS.

Methods 29 SLE patients entered the study, 13 had secondary APS $(\mathrm{M} / \mathrm{F}=1 / 12$; mean age $=45.5 \mathrm{yrs}$; mean disease duration $=5.85 \mathrm{yrs})$ while the other 16 patients $(\mathrm{M} / \mathrm{F}=1 / 15$; mean age $=36.6 \mathrm{yrs}$; mean disease duration $=7.2 \mathrm{yrs}$ ) had no clinical or laboratory signs of APS. There were no significant differences regarding the number of ARA criteria or ECLAM and SLEDAI scores between the two groups.

Each patient underwent nailfold capillaroscopy to detect the following abnormalities: morphological alterations of capillaries, variability of capillary loop lenght, presence of microhaemorrages, irregular distribution of capillaries and sludging of blood. Moreover to score these changes a semiquantitative rating scale was adopted, according to previous studies.

Results Although not statistically significant, more frequent variability of capillary loop lenght ( $77 \%$ vs $44 \%)$, presence of microhaemorrages $(23 \%$ vs $12.5 \%)$, sludging of blood $(23 \%$ vs $12.5 \%)$ as well as capillaroscpopic score $>1$ (77\% vs $44 \%)$ were found in patients with SLE/APS respect to those with SLE alone.

However in our SLE/APS patients the presence of capillaroscopic microhaemorrages was significantly associated to thrombocytopenia $(p<0.034)$ and the finding of shorter capillary 